\title{
Research on the Effects of Mechanical and Physical Characteristics on Peanut Shucking
}

\author{
Hongmei Xu, Shuiping Yan, and Qi Huang \\ College of Engineering, Huazhong Agriculture University, Wuhan 430070, China \\ xhm790912@163.com
}

\begin{abstract}
Shucking is one of the necessary parts for the deep process of peanut, and has great impact on the quality of peanut kernel.Taking the shucking force as the evaluation indicator, the effects of mechanical and physical characteristics, which include the loading orientation, loading rate, moisture content, partical size and the number of peanut kernel, on peanut shucking were investigated by means of single factor test. The results show that: 1) the loading orientation and moisture content affect the shucking force significantly, and the needed force is the minimum when the peanut is vertically placed and loaded. With the decrease of moisture content, the needed force decreases gradually.2) the loading rate, particle size and the number of peanut kernel have no great effect on the shucking force, but the deformation and maximum shucking force vary with the loading rate. Additionally, the needed force increases with the number of peanut kernel. The research results can provide some reference for the designment of peanut sheller and selection of the technique parameters.
\end{abstract}

Keywords: Peanut, Shucking, Mechanical Characteristic, Physical Property.

\section{Introduction}

In recent years, with the great increase of peanut yield, the peanut foods have developed in the direction of deep processing. Shucking is one of the necessary steps for the deep process of peanut, which usually results in the damage and crack, and determines the quality of peanut kernel [1]. Nowadays, because of the unreasonable force-applying forms, most peanut shellers used in domestic are of larger breaking rate and behave themselves with poor shucking quality [2].

Mechanical and physical characteristics can provide fundamental data for developing the sheller and realizing the effective separation of peanut shell and meat. In order to design the highly effective and low damage sheller, mechanical and physical characteristics of peanut must be first investigated.

Take the peanut of E-hua NO.6 as the research object, and the compression test was conducted on the universal material testing machine. The effects of the loading orientation, loading rate, moisture content, partical size and the number of peanut kernel on the peanut shucking force were investigated by means of single factor test. The research results are of great significance for improving the working performance of peanut sheller and designing the new and high-efficiency shucking equipments. 


\section{Materials and Methods}

\subsection{Materials}

The sold E-hua NO.6 peanut was selected as the specimen, and the experiment has been finished in the comprehensive laboratory of College of Engineering, Huazhong Agriculture University, in May 2011.

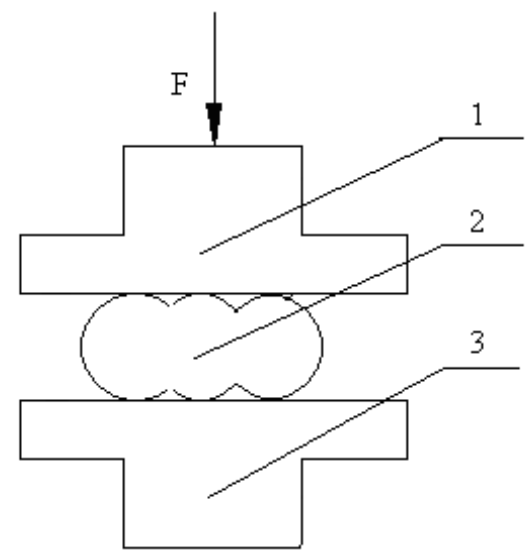

\section{Upper platen $\quad$ 2.Specimen $\quad 3$. Bottom platen}

Fig. 1. Sketch of the loading device

\subsection{Devices}

The experiment was conducted on the universal material testing machine of RGT2000-10, and the loading rate varies from $0.01 \mathrm{~mm} / \mathrm{min}$ to $500 \mathrm{~mm} / \mathrm{min}$. Fig.1 shows the sketch of the loading device. The pressure imposed on the peanut specimen by the plate-type pressure head is measured by the force transducer.

\subsection{Methods}

The experiment was conducted on the universal material testing machine, and the specimen was applied with the static load. In the beginning of the experiment, put the specimen on the bottom platen of the testing machine, and adjust the location of the upper platen to make sure it contacts with the specimen and imposes a compression force on the specimen. In order to determine the influence of the mechanical and physical characteristics on peanut shucking, the single factor test was conducted to investigate the effects of the loading orientation, loading rate, moisture content, particle size and the number of peanut kernel on the peanut shucking force. Additionally, for each experimental condition, the experiment was repeated ten times to reduce the test tolerance, and the result is the mean value of 10 tests. 


\section{Results and Discussion}

\subsection{Analysis of the Cracking Process of Peanut Shell}

\subsubsection{Cracking Forms of Peanut Shell}

Fig. 2 shows the cracking forms of peanut shell when the peanuts are applied with loads in different directions. The cracking forms of peanut shell are of two main types, namely, cracking longitudinally and cracking transversely. Because of the structural inhomogeneity and discontinuity of the peanut ridge, it features low compression strength. When the peanut is horizontally placed and applied with a load, the stress concentration and longitudinal crack usually occur. The longitudinal crack propagates in the direction of the peanut ridge, and plays an important role in the peanut shucking. When the peanut is vertically placed and applied with a load, the cracking form is similar to that of peanut shell when it is horizontally placed. The crack mainly concentrates on the end and ridge of peanut. However, when the peanut is placed on its side, as shown in Fig.2 (b), and applied with a load, the transverse crack occurs, and the peanut cracks from the loading point out to the outward, which usually brings about a depression in the loading point [3]

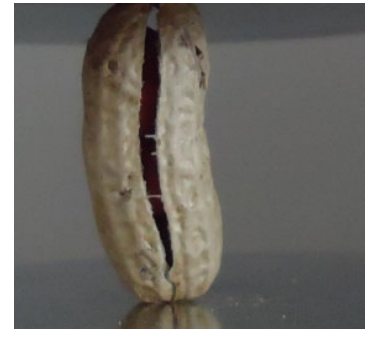

(a) Cracking longitudinally

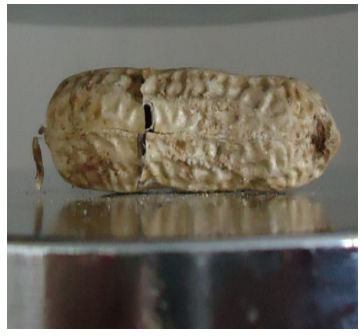

(b) Cracking transversely

Fig. 2. Crack propagation of peanut shell

\subsubsection{Cracking Process of Peanut Shell}

Fig. 3 shows the relation curve between the loading force and deformation of peanut when it is horizontally placed and applied with a load. As shown in the figure, the cracking process of peanut shell mainly includes three stages, namely, elastic deformation, plastic deformation and sudden rupture. The elastic deformation mainly refers to the early loading stage, in which the pressure linearly increases with the deformation of peanut. Afterwards, with the increase of loading force, the elastic deformation goes hand in hand with plastic deformation. The plastic deformation mainly focuses in the later loading stage. However, when the loading force achieves the peak value for the first time, the peanut begins to crack and the force declines sharply. The peak value mentioned above mainly depends on the compressive strength of peanut, so it's also known as the peanut shucking force. Then, the peanut shell and meat is compressed together, and the required loading force increases again $[3,4]$. In the course of the experiment, in order to protect the testing machine, the experiment is called for a halt once the peanut begins to crack and the loading force achieves the maximum. 


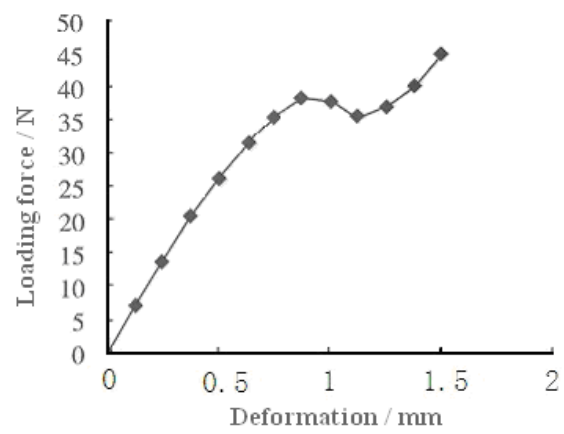

Fig. 3. Relation curve between the loading force and deformation of peanut

\subsection{Analysis of the Influence Factors of Peanut Cracking}

\subsubsection{Effects of Loading Orientation on Peanut Cracking}

Select thirty medium-size dried peanuts as the specimen, which is coincided with each other in the size and moisture content, and each peanut contains three kernels. Divide the peanuts into three groups, and each group contains ten peanuts. In the course of the experiment, the ten peanuts in group 1 are all horizontally placed as shown in Fig. 4(a).The peanuts in group 2 and group 3 are placed on their sides and vertically placed respectively. Compress the three groups of specimens at the loading rate of $10 \mathrm{~mm} / \mathrm{min}$, and don't cease the experiment until the peanut shell begins to crack, which results in the great reduction of pressure. Record the peak value of the pressure, and regard it as the peanut shucking force.
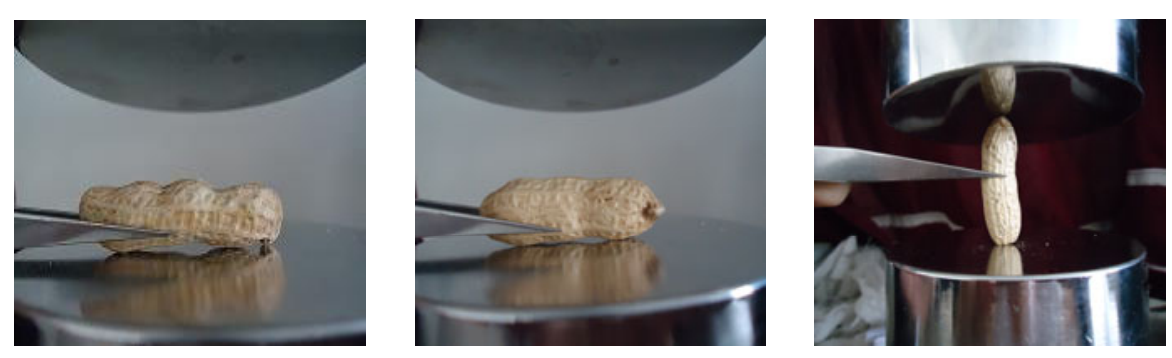

(a) Peanut is horizontally placed (b) Peanut is placed on its side (c) Peanut is vertically placed

Fig. 4. Loading orientation of Peanut

Fig.5 shows the testing results of the peanut shucking force when the peanuts are applied with loads in different directions. As shown in the figure, the needed shucking forces are clearly different when the peanuts are applied with loads in different directions. When the peanut is vertically placed, the needed force is the minimum, which is equal to $19.3 \mathrm{~N}$, and then is the needed force when the peanut is horizontally placed. The needed force is the maximum when the peanut is placed on its side. The reason is that the load is mainly concentrated on the end and ridge of peanut when the 
peanut is vertically placed and applied with a load. Because of the smaller stressed area, the stress is larger, which makes the peanut crack easily. When the peanut is horizontally placed, the load mainly acts on the ridge of peanut. Because of the structural inhomogeneity and discontinuity of the peanut ridge, the stress concentration and longitudinal crack with definite direction usually occurs, which contributes much to shell shucking. However, when the peanut is placed on its sides, the load is mainly concentrated on the middle part of peanut, and the larger stressed area results in smaller stress, which works against the shell shucking of peanut [4]. Variance analysis was conducted for the testing results of peanut shucking force, and the corresponding F-statistic is equal to 77.26, which is greater than the critical value $F_{\alpha}(2,27)(\alpha=0.05)$.The variance analysis results are consistent with the testing results. It is, therefore, believed that the loading orientation has great impact on the peanut shucking force.

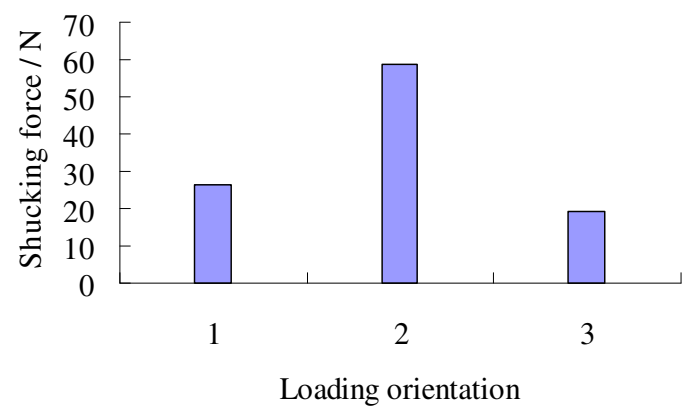

1: Peanut is horizontally placed

2: Peanut is placed on its side

3: Peanut is vertically placed

Fig. 5. Shucking force of peanut with different loading orientation

\subsubsection{Effects of Loading Rate on Peanut Cracking}

Select forty medium-size dried peanuts as the specimen, which is coincided with each other in the size and moisture content, and each peanut contains three kernels. Divide the peanuts into four groups, and each group contains ten peanuts. In the course of the experiment, the forty peanuts are all horizontally placed. Compress the four groups of specimens at the loading rate of $10 \mathrm{~mm} / \mathrm{min}, 20 \mathrm{~mm} / \mathrm{min}, 30 \mathrm{~mm} / \mathrm{min}$ and $40 \mathrm{~mm} / \mathrm{min}$ respectively.

Fig. 6 shows the testing results of the peanut shucking force when the peanuts are compressed at different loading rates. As shown in the figure, when the peanuts are compressed at different loading rates, the needed shucking forces are different, but the difference is not large. When the peanut is compressed at the loading rate of $10 \mathrm{~mm} / \mathrm{min}$, the needed force is the minimum, which is equal to $26.1 \mathrm{~N}$, and then is the loading rate of $20 \mathrm{~mm} / \mathrm{min}$. The needed force is the maximum when the peanut is compressed at the loading rate of $30 \mathrm{~mm} / \mathrm{min}$ and $40 \mathrm{~mm} / \mathrm{min}$. Variance analysis was conducted for the testing results of peanut shucking force when the peanuts are 
compressed at different loading rates, and the corresponding F-statistic is equal to 0.44 , which is less than the critical value $F_{\alpha}(4,35)(\alpha=0.05)$. So it can be concluded that the loading rate has no great effect on the peanut shucking force.

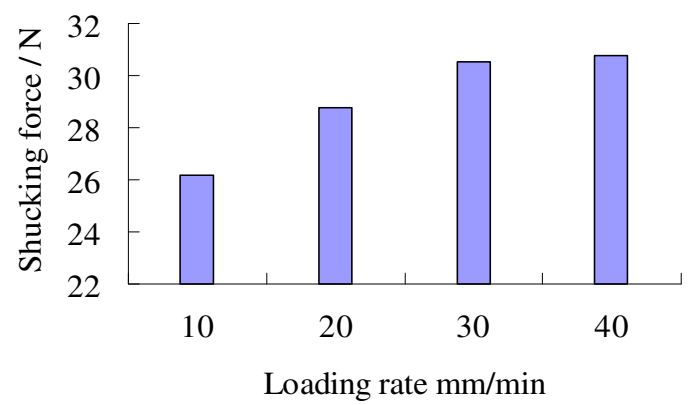

Fig. 6. Shucking force of peanut with different loading rate

\subsubsection{Effects of Particle Size on Peanut Cracking}

Select thirty dried peanuts as the specimen, which is coincided with each other in the moisture content, and each peanut contains three kernels. Divide the peanuts into three groups, and each group contains ten peanuts. The ten peanuts in group 1 with larger size range from $19 \mathrm{~mm}$ to $21 \mathrm{~mm}$ in diameter. The ten peanuts in group 2 are of medium size, and range from $14 \mathrm{~mm}$ to $16 \mathrm{~mm}$ in diameter. The ten peanuts in group 3 are the smallest ones, and range from $9 \mathrm{~mm}$ to $11 \mathrm{~mm}$ in diameter. In the course of the experiment, the thirty peanuts are all horizontally placed, and compressed at the loading rate of $10 \mathrm{~mm} / \mathrm{min}$.

Fig.7 shows the testing results of the shucking force of peanut with different particle size. For the peanuts with different particle size, the needed forces are different, but the difference is not large. The needed shucking force of the large-size peanuts is the minimum, which is equal to $24.6 \mathrm{~N}$, and then is the smaller ones.

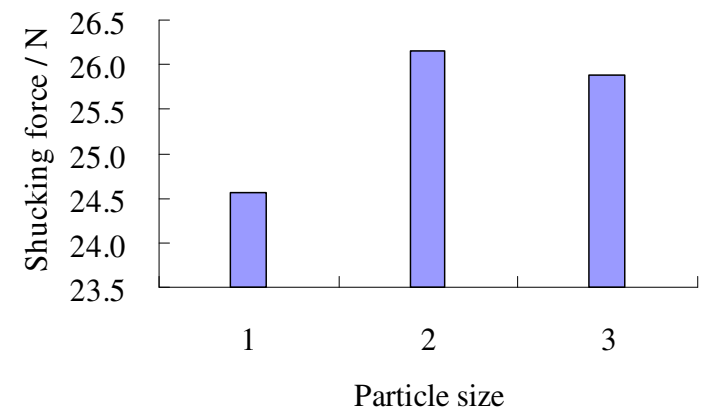

1: Peanut is of larger size

2: Peanut is of medium size

3: Peanut is of smaller size

Fig. 7. Shucking force of peanut with different particle size 
The needed shucking force of the medium-size peanuts is the maximum. Variance analysis was conducted for the testing results of the shucking force of peanut with different particle size, and the corresponding F-statistic is equal to 0.15 , which is less than the critical value $F_{\alpha}(2,27)(\alpha=0.05)$. It can be concluded that particle size has no great effect on the peanut shucking force.

\subsubsection{Effects of Peanut Kernel Number on Peanut Cracking}

Select forty dried peanuts as the specimen, which is coincided with each other in the moisture content. Divide the peanuts into four groups, and each group contains ten peanuts. The peanuts in group 1 and group 2 contain one and two kernels respectively. The peanuts in group 3 and group 4 contain three and four kernels respectively. In the course of the experiment, the four groups of peanuts are all horizontally placed, and compressed at the loading rate of $10 \mathrm{~mm} / \mathrm{min}$. Fig. 8 shows the testing results of the shucking force of peanut with different kernel number. As shown in the figure, different kernel number corresponds to different shucking force, but the difference is not large. The needed shucking force of the one-kernel peanuts is the minimum, which is equal to $21.3 \mathrm{~N}$.Furthermore, the needed force increase with the increase of the peanut kernel number. The needed shucking force of the fourkernel peanuts is the maximum. Variance analysis was conducted for the testing results of the shucking force of peanut with different kernel number, and the corresponding F-statistic is equal to 2.19 , which is less than the critical value $F_{\alpha}(3,36)(\alpha=0.05)$. It can be concluded that kernel number has no great effect on the peanut shucking force.

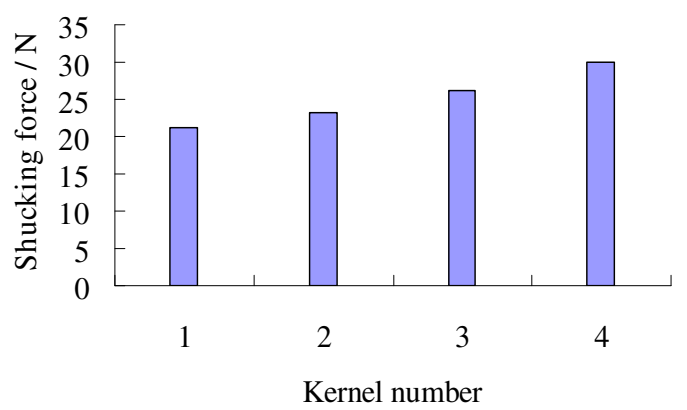

Fig. 8. Shucking force of peanut with different kernel number

\subsubsection{Effects of Moisture Content on Peanut Cracking}

Select fifty medium-size dried peanuts as the specimen, and each peanut contains three kernels. Divide the peanuts into five groups, and each group contains ten peanuts. In order to investigate the effect of the moisture content on the peanut shucking force, the peanuts must be pretreated before the compression test. The drying experiment is one of the most important preprocessing methods, which is usually used to control the moisture content of the samples. In this research, the drying experiments were conducted for five groups of walnut specimens, and the 
corresponding drying time is $0 \mathrm{~h}, 0.5 \mathrm{~h}, 1 \mathrm{~h}, 1.5 \mathrm{~h}$ and $2 \mathrm{~h}$ respectively. Afterwards, the peanuts are all horizontally placed, and compressed at the loading rate of $10 \mathrm{~mm} / \mathrm{min}$.

Fig.9 shows the testing results of the shucking force of peanut with different drying time and moisture content. As shown in the figure, different drying time corresponds to different peanut shucking force. When the drying time is $2 \mathrm{~h}$, the needed shucking force is the minimum, which is equal to $21.9 \mathrm{~N}$, and then is the peanuts with the drying time of $1 \mathrm{~h}$ and $1.5 \mathrm{~h}$. The needed shucking force of the peanuts with the drying time of $\mathrm{Oh}$ is the maximum. It can be seen that the moisture content of peanuts decreases with the increase of drying time. As a result, the needed shucking force decreases which makes peanut shucking easier. The reason is that the lower moisture content corresponds to greater brittleness, which brings about the decrease of the peanut antiforce capability [5].Variance analysis was conducted for the testing results of the shucking force of peanuts with different moisture content, and the corresponding F-statistic is equal to 10.72 , which is greater than the critical value $F_{\alpha}(4,45)$ $(\alpha=0.05)$. Therefore, it can be concluded that the drying time and moisture content affect the peanut shucking force significantly.

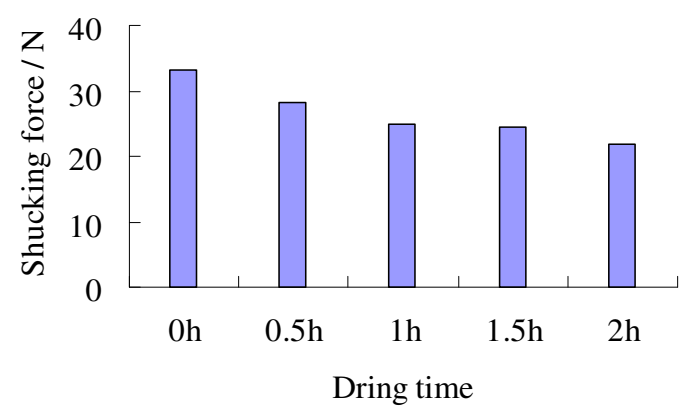

Fig. 9. Shucking force of peanut with different moisture content

\section{Conclusions}

Take the shucking force as the evaluation indicator, and the effects of the loading orientation, loading rate, moisture content, particle size and peanut kernel number on peanut shucking were investigated by means of single factor test. The conclusions can be described as follows:

(1) Loading orientation has great impact on the peanut shucking force. When the peanuts are vertically placed, the needed shucking force is the minimum, and then is the peanuts horizontally placed. The needed shucking force of the peanuts placed on their sides is the maximum.

(2) Moisture content affects the peanut shucking force significantly, and with the increase of drying time, the moisture content of peanut decrease gradually, which results in the decreases of the needed shucking force. 
(3) Loading rate, particle size and number of peanut kernel have no great effect on the shucking force, but different loading rate and particle size correspond to different shucking force. Additionally, the needed force increases with the number of peanut kernel.

(4) The cracking forms of peanut shell are of two main types, namely, cracking longitudinally and cracking transversely. When the peanuts are horizontally and vertically placed and applied with a load, the longitudinal crack usually occurs, which makes peanut shucking easier. The transverse crack occurs only when the peanuts are placed on their sides.

Acknowledgement. This research was supported by Student's Science \& Technology Innovation Fund of Huazhong Agricultural University "Research on the prediction of the acoustic performance for vehicle-use sound packages based on FE-SEA hybrid model" under grant Nos. 11028.

\section{References}

1. Shuqi, S., Shuguang, L., Fangyan, W.: Current Situation and Development of Peanut Production Machinery. Transactions of the Chinese society for Agricultural Machinery 36(3), 143-147 (2005)

2. Jiandong, L., Shuqi, S., Xizhen, L., et al.: Current Situation and Development of Peanut Shelling Machine and Equipment in China. Journal of Peanut Science 35(4), 23-27 (2006)

3. Hongli, L., Yongli, Z., Lianxing, G., et al.: Experimental Research of the Shelling Force Performance of Groundnuts. Journal of Shenyang Agricultural University 37(6), 900-902 (2006)

4. Lihua, Z., Wen, Z., Wen, Q., et al.: Mechanical Characteristics of Peanut Cracking. Food Science 31(13), 52-55 (2010)

5. Xianli, C., Lianxing, G., Mingguo, L., et al.: Experimental Study on Peanuts Mechanical Performance under Impacting Test. Journal of Shenyang Agricultural University 40(1), 111-113 (2009) 